\title{
Medication Adherence Patterns Among Patients with Multiple Serious Mental and Physical Illnesses
}

Joanna P. MacEwan - Alison R. Silverstein · Jason Shafrin •

Darius N. Lakdawalla · Ainslie Hatch · Felicia M. Forma

Received: March 6, 2018 / Published online: May 3, 2018

(C) The Author(s) 2018

\begin{abstract}
Introduction: Patients with mental and physical health conditions are complex to treat and often use multiple medications. It is unclear how adherence to one medication predicts adherence to others. A predictive relationship could permit less expensive adherence monitoring if overall adherence could be predicted through tracking a single medication.
\end{abstract}

Enhanced digital content To view enhanced digital content for this article go to https://doi.org/10.6084/ m9.figshare.6122075.

Electronic supplementary material The online

version of this article (https://doi.org/10.1007/s12325-

018-0700-6) contains supplementary material, which is available to authorized users.

J. P. MacEwan $(\bowtie) \cdot$ A. R. Silverstein · J. Shafrin Precision Health Economics, 11100 Santa Monica Blvd, Suite 500, Los Angeles, CA 90025, USA e-mail:

Joanna.macewan@precisionhealtheconomics.com

D. N. Lakdawalla

Schaeffer Center for Health Policy and Economics, University of Southern California, Los Angeles, CA 90089, USA

A. Hatch

Otsuka America Pharmaceutical, Inc., 508 Carnegie Center Drive, Princeton, NJ 08540, USA

F. M. Forma

Otsuka Pharmaceutical Development \& Commercialization, Inc., 508 Carnegie Center

Drive, Princeton, NJ 08540, USA
Methods: To test this hypothesis, we examined whether patients with multiple mental and physical illnesses have similar adherence trajectories across medications. Specifically, we conducted a retrospective cohort analysis using health insurance claims data for enrollees who were diagnosed with a serious mental illness, initiated an atypical antipsychotic, as well as an SSRI (to treat serious mental illness), biguanides (to treat type 2 diabetes), or an ACE inhibitor (to treat hypertension). Using group-based trajectory modeling, we estimated adherence patterns based on monthly estimates of the proportion of days covered with each medication. We measured the predictive value of the atypical antipsychotic trajectories to adherence predictions based on patient characteristics and assessed their relative strength with the $R$ squared goodness of fit metric.

Results: Within our sample of 431,591 patients, four trajectory groups were observed: non-adherent, gradual discontinuation, stopstart, and adherent. The accuracy of atypical antipsychotic adherence for predicting adherence to ACE inhibitors, biguanides, and SSRIs was $44.5,44.5$, and $49.6 \%$, respectively (all $p<0.001$ vs. random). We also found that information on patient adherence patterns to atypical antipsychotics was a better predictor of patient adherence to these three medications than would be the case using patient demographic and clinical characteristics alone. 
Conclusion: Among patients with multiple chronic mental and physical illnesses, patterns of atypical antipsychotic adherence were useful predictors of adherence patterns to a patient's adherence to ACE inhibitors, biguanides, and SSRIs.

Funding: Otsuka Pharmaceutical Development \& Commercialization, Inc.

Keywords: Adherence; Atypical anti-psychotic; Neurology; Serious mental illness; Trajectory model

\section{INTRODUCTION}

Patients with serious mental illness (SMI), approximately $4 \%$ of US adults [1], are at higher risk than the general population for chronic physical comorbidities [2-6]. Patients with depression are 1.5 times more likely to have cardiovascular disease. Patients with schizophrenia or bipolar disorder are 2-3 times more likely to have cardiovascular disease. Patients with schizoaffective or bipolar disorder are 2-3 times more likely to have diabetes mellitus [7]. These patients with SMI and comorbid physical conditions are prescribed multiple medications to treat their symptoms and manage their conditions [8], and medication nonadherence is common.

Addressing these adherence issues is complex, particularly for patients prescribed multiple medications for their SMI and comorbid chronic physical conditions [2-6]. Estimates of adherence to atypical antipsychotics for schizophrenia and related disorders, for example, range from $47 \%$ to $95 \%$ [9], indicating both low rates of adherence and potentially inaccurate or insufficient measurements of adherence. Rates of medication adherence are further reduced as the number of medications prescribed increases [10, 11]. Medication non-adherence among patients with SMI contributes to higher rates of relapse [12, 13], hospitalization [14-17]. increased risk of mortality [18], and lower health-related quality of life [19]. Costs of treatment for SMI alone exceed US\$300 billion annually to the healthcare system [20], while hospitalizations due to medication non- adherence cost more than $\$ 100$ billion annually [21]. These cost estimates would rise further if we accounted for the indirect costs of incarceration, homelessness, and other adverse events due to mental and physical comorbid conditions and non-adherence [20].

Commonly-used adherence measures do not fully capture the adherence patterns of patients with serious mental and physical illnesses. Health insurance claims data only track medication fills, not ingestion, and typically measure adherence using a single metric of average adherence over a period of time [22]. Proportion of days covered (PDC) is a commonly used secondary analysis method to describe the proportion of days during a specified period that the patient is in possession of the medication [23]. While prominent organizations such as the International Society for Pharmacoeconomics and Outcomes Research and the National Committee for Quality Assurance endorse PDC as a preferred method of calculating medication adherence $[24,25]$, there are limitations to this approach. Metrics such as PDC do not capture variation in adherence over time [26]. For instance, there may be two patients with PDC values of $50 \%$, but the first may use the medication every other month while the second may be perfectly adherent for 6 months and then discontinue. Despite having identical PDC values, for the first patient with intermittent adherence, case management or adherence interventions may be more appropriate; for the latter patient who discontinued treatment, physicians may need to try new medications to deal with potential adverse events.

Despite the limitations of using PDC to measure adherence, physician access to quantitative data on patient adherence is often more limited. Physicians often rely on patient selfreports to measure adherence, but self-reported adherence is biased due to social stigma and memory issues [27], leading to poor concordance between patient self-reported adherence and objective measures of adherence [28]. Overall, physician's assessment of patient's adherence levels is generally not much better than a random guess (53 vs. 50\%) [29]. 
In this paper, we rely on group-based trajectory modeling to examine whether patients with multiple serious mental and physical illnesses have similar adherence trends across all their treatments [26]. Group-based trajectory modeling provides a data-driven method for identifying groups of patients with different trends and trajectories in adherence behavior. In our example above, group-based trajectory modelling is able to separately identify clusters of patients who are irregularly adherent throughout the year from those who discontinue therapy, even if these clusters have similar PDC levels.

In this study, we employ this method to analyze adherence behavior for patients with schizophrenia, bipolar disorder, or major depressive disorder initiating atypical antipsychotic therapy and concurrently prescribed medication for another SMI, type 2 diabetes, or hypertension. We test whether adherence patterns for the atypical antipsychotic mirror adherence patterns for other SMI, anti-diabetes, and anti-hypertension medications. To our knowledge, this is the first time the trajectory modeling approach has been applied to examine adherence patterns for multiple drug classes simultaneously.

\section{METHODS}

We conducted a retrospective claims analysis using data from Truven MarketScan ${ }^{\circledR}$ Commercial Claims and Encounters Database, Medicare Supplemental Claims and Encounters Database, and Medicaid Multi-state Database, all from January 1, 2008 through December 31, 2014. The commercial claims include medical and pharmacy claims from employer-sponsored plans for 8 million enrollees and their dependents. The Medicare database provides the Medicare Supplemental data and includes medical and pharmacy claims for 13.3 million people enrolled in a Medicare Advantage and/or a Medicare Part D plan. The Medicaid claims represent medical and pharmacy claims from 1.2 million subscribers from 11 states. This article does not contain any studies with human participants or animals performed by any of the authors.

Our sample of patients included patients with serious mental illness who initiated an atypical antipsychotic. In order to be included in the study, patients had to be age 18 years or older at the index date. They had to have $\geq 1$ inpatient claim or $\geq 2$ outpatient claims with a diagnosis of schizophrenia (ICD-9-CM 295.x) [30], bipolar disorder (ICD-9-CM 296.0x-296.1x, 296.4x296.8x) [34, 35], or major depressive disorder (ICD-9-CM 296.2x, 296.3x, 311.x) [32, 33], 6 months prior to initiating an antipsychotic. From this set of patients, we created three patient cohorts: (1) patients with SMI who also initiated selective serotonin reuptake inhibitors (SSRIs), (2) patients with SMI and type 2 diabetes who also initiated treatment with biguanides, and (3) patients with SMI and hypertension who also initiated an angiotensin-converting enzyme (ACE) inhibitor. We identified patients with type 2 diabetes (ICD-9-CM 250.00, 250.01, 250.02, 250.03, 790.2x, 791.5, 791.6, V45.85, V53.91, V65.46) [33, 34] or hypertension (ICD-9-CM 401.9, 401.0, 402.x, 403.x, 404.x, 405.01, 405.09, $405.11,405.19,405.91,405.99,437.2)$ based on the relevant ICD-9-CM codes during the 6 months prior to (and including) the date of treatment initiation. For all three cohorts, patients were required to be continuously enrolled 6 months prior to and 12 months after the first observed antipsychotic fill and the first observed fill for another SMI, anti-diabetes, or anti-hypertension medication in the data. Patients who received their atypical antipsychotics through mail order were excluded in order to decrease the possibility of overestimating adherence levels due to automatic refills by mail. Patients were also excluded if they had an index fill for clozapine. Consistent monitoring and physician supervision is required for clozapine use [35], which may impact prescription refill and adherence rates [36].

The selections of SSRIs, ACE inhibitors, and biguanides as case studies for the treatment of other SMI, type 2 diabetes, and hypertension, respectively, were based on clinical guidelines, published literature, expert clinician recommendations and sample size considerations. SMI treatments were informed by 
recommendations from the American Psychiatric Association Clinical Practice Guidelines [40-44]. Anti-diabetes and anti-hypertension medications were selected from clinical literature [45-48]. For the purposes of this study, a number of atypical antipsychotic medications were included to ensure that we could maintain a pattern of adherence even if a patient switched atypical antipsychotic molecules. These medications were risperidone, olanzapine, quetiapine, ziprasidone, aripiprazole, lurasidone, paliperidone, iloperidone, and asenapine. Specific molecules included in each class of medication selected can be found in Online Resource Table A1.

For each of the three cohorts determined by medication pairs, we used group-based trajectory models to measure adherence patterns over time. To create these trajectories, we first measured adherence to each treatment for the 12 months following treatment initiation. We defined patients as adherent in any given month if $\mathrm{PDC} \geq 80 \%[46,47]$. After creating a series of 12 indicator variables defining whether each patient was adherent to the relevant medications, we applied the group-based trajectory model algorithm to simultaneously sort individuals into adherence groups based on monthly PDC to both the index atypical antipsychotic and the index medication to treat other SMI, diabetes, or hypertension. Study authors have applied a similar approach to measuring adherence trends among patients with schizophrenia in previous research [26], and a similar algorithm was used here.

More specifically, the trajectory model was estimated in Stata-MP 13.0 using the user written traj command/program. Within each adherence trajectory group $j$, adherence to medication $k, A_{\mathrm{k}}$, was modeled as a smooth function of time (measured in months elapsed since the index date), $t$, using a third-degree polynomial. We modeled the adherence trajectory as a generalized logit model that allows time-invariant patient characteristics to influence the probability of group membership:

$$
\operatorname{Pr}\left(A_{i, k} \mid t_{i}\right)=\sum_{j=1}^{J} \pi_{j}\left(x_{i}\right) \operatorname{Pr}\left(A_{i, k} \mid \boldsymbol{t}_{i}, j ; \boldsymbol{\beta}_{j, k}\right),
$$

where

$$
\pi_{j, k}\left(x_{i}\right)=\frac{e^{x_{i} \theta_{j, k}}}{\sum e^{x_{i} \theta_{j, k}}} .
$$

The parameter $\pi_{j, k}$ represents the probability of membership to group $j$ (an estimated model parameter) for drug $k$, which depends on timeinvariant individual characteristics $x_{i}$, and the elements of vector $\boldsymbol{\beta}_{\boldsymbol{j}, \boldsymbol{k}}$ are the estimated coefficients on the month variables $\boldsymbol{t}_{\boldsymbol{i}}$. We compared group-based trajectory model results using four adherence trajectory groups. In this application, the vector of patient characteristics $x_{i}$ included only a constant term since we were interested in the relationship between adherence patterns across a patient's therapies rather than controlling for specific factors that could predict different adherence patterns. For each of the trajectory groups formed, we compared antipsychotic adherence trajectories to those of SSRIs, ACE inhibitors, and biguanides using a visual predictive check.

Once the trajectory groups were formed, our first research question addressed how well patients' antipsychotic adherence patterns could predict their patterns of adherence to their other medications (i.e., SSRI, ACE inhibitor, or biguanides). To study this, we first assigned patients to the trajectory group to which they had the highest probability of belonging. Next, we measured the conditional probability that patients were in the same trajectory group for non-antipsychotic medications, conditional on their placement in that particular atypical antipsychotic trajectory group. Among patients using antipsychotics and ACE inhibitors, for instance, if a patient was in a consistently adherent trajectory for atypical antipsychotics use, we measured the likelihood that the patient was also in the consistently adherent trajectory group for their ACE inhibitor. We followed this procedure for all four adherence groups, and then we measured the overall likelihood that the patient's assigned atypical antipsychotic adherence trajectory 
could predict their adherence to their ACE inhibitor. A similar approach was used for all three patient cohorts. We then conducted a $t$ test to estimate whether the share of patients whose antipsychotic adherence trajectory matched their adherence trajectory for other medications was different from random chance (i.e., $25 \%$ in the four-trajectory case).

We investigated a second research question comparing the predictive value of these adherence trajectories to adherence predictions based on patient characteristics. We estimated a logistic regression model for each adherence trajectory for the "other" medication (i.e., ACE inhibitor, biguanides, SSRI) first using only the indicator variables for the antipsychotic adherence trajectory to which they were assigned. We then replicated this same logistic regression, but rather than including atypical antipsychotic adherence patterns as the explanatory variables, we included patient demographics (age, gender, region, Charlson Comorbidity Index, and index antipsychotic used). We estimated this logistic regression a third time combining adherence trajectories and patient demographics. We calculated the relative strength of these relationships using the $R$-squared goodness of fit metric. Accuracy was measured using the $R$-squared metric.
One limitation of applying the group-based trajectory model was that the number of adherence trajectories needed to be pre-specified. To test how our results would vary based on the number of trajectory groups selected, we replicated the results with three groups in a sensitivity analysis.

\section{RESULTS}

\section{Patient Sample}

We used a sample of 436,591 patients, consisting of 241,648 commercially insured enrollees, 168,411 Medicaid enrollees, and 26,502 Medicare enrollees (Table 1). The patient sample is described in Table 2 . The mean ages by payer type were 40.9 years among commercially insured beneficiaries, 41.7 years within Medicaid, and 76.1 years for those with Medicare. Our sample was majority female $(63.7 \%$ in commercial, $65.8 \%$ in Medicaid, and $65.4 \%$ in Medicare). Medicare patients had more Charlson comorbidities (1.43) compared to other types of enrollees (0.30 commercial, 0.60 Medicaid). The greatest proportion of patients in Medicare and Medicaid had major depressive disorder diagnosis (58.6 and $46.5 \%$, respectively). In the commercially insured group with

Table 1 Patient cohorts, by payer

\begin{tabular}{|c|c|c|c|}
\hline & Commercial & Medicare & Medicaid \\
\hline Enrolled patients & $11,053,733$ & $1,229,651$ & $2,791,550$ \\
\hline$\geq 18$ years of age & $10,586,378$ & $1,229,651$ & $2,415,776$ \\
\hline Received oral atypical antipsychotic & 919,872 & 136,038 & 496,092 \\
\hline $\begin{array}{l}\text { Enrolled continuously } 6 \text { months prior and } 12 \text { months after first oral atypical } \\
\text { and } \geq 18 \text { in year indexed }\end{array}$ & 366,234 & 62,946 & 210,367 \\
\hline Exclude if antipsychotic through mail order & 304,118 & 42,097 & 207,149 \\
\hline Exclude if index fill for clozapine & 303,157 & 41,945 & 204,130 \\
\hline Has claims during atypical enrollment window & 303,157 & 41,945 & 204,130 \\
\hline $\begin{array}{l}\geq 1 \text { inpatient or } \geq 2 \text { outpatient claims with a diagnosis code for SCZ, BPD, or } \\
\quad \text { MDD during } 6 \text { months prior to index date or } 12 \text { months after }\end{array}$ & 241,648 & 26,502 & 168,441 \\
\hline
\end{tabular}

$S C Z$ schizophrenia, $B P D$ bipolar disorder, $M D D$ : major depressive disorder 
atypical antipsychotic prescription fills, $29.0 \%$ had a major depressive disorder diagnosis and $30.5 \%$ had a schizophrenia diagnosis.

\section{Trajectory Analysis}

In our baseline analysis, groups were defined as non-adherent, gradual discontinuation, stopstart, and adherent. These patterns can be seen in Fig. 1, depicting the trajectories for ACE inhibitors (Fig. 1a), biguanides (Fig. 1b), and SSRIs (Fig. 1c). In the non-adherent group, patients immediately discontinued treatment, and by month 3 had a probability of adherence approximately $20 \%$ or less across all medication combinations. The gradual discontinuation group generally maintained adherence for 3-5 months, but dropped below a 50\% probability of adherence between months 6 and 7, and were less than 30\% adherent by month 9 . In the stop-start group, adherence decreased to almost $30 \%$ by month 4 but increased again to reach $70-80 \%$ between months 9 and 12 . Finally, the adherent group maintained a probability of adherence between 90 and 100\% during the entire 12-month period. A visual predictive check confirmed that the
Fig. 1 Four-group adherence trajectories for atypical antipsychotics and (a) ACE inhibitors, (b) biguanides, and (c) SSRIs

antipsychotic trajectory groups were qualitatively and quantitatively similar to the trajectories for the three comparison drug classes.

Similar proportions of patients using an ACE inhibitor or an SSRI were observed in each adherence trajectory group, but the adherence patterns were distinct among those who took biguanides. Patients with SMI and hypertension taking an atypical antipsychotic and an ACE inhibitor were distributed across the adherence groups similarly between their medications. Atypical antipsychotic adherence patterns in this patient population were $25.1 \%$ non-adherent, $13.8 \%$ gradual discontinuers, $11.5 \%$ stopstart, and $49.6 \%$ adherent. The distribution of ACE inhibitor adherence groups was 26.5\% non-adherent, $15.0 \%$ gradual discontinuers, $11.7 \%$ stop-start, and $46.8 \%$ adherent. The population of patients with SMI taking an atypical antipsychotic and an SSRI was also observed to be similarly distributed across trajectory groups in both medications. The distribution of adherence to atypical antipsychotics

Table 2 Patient characteristics, by payer

\begin{tabular}{lccc}
\hline & $\begin{array}{l}\text { Commercial } \\
(\boldsymbol{n}=\mathbf{2 4 1 , 6 4 8})\end{array}$ & $\begin{array}{l}\text { Medicare } \\
(\boldsymbol{n}=\mathbf{2 6 , 5 0 2})\end{array}$ & $\begin{array}{l}\text { Medicaid } \\
(\boldsymbol{n}=\mathbf{1 6 8 , 4 4 1})\end{array}$ \\
\hline Age, mean (SD) & $40.9(13.25)$ & $76.07(9.41)$ & $41.674(13.94)$ \\
Female, $n$ (\%) & $153,906(63.69 \%)$ & $17,333(65.40 \%)$ & $110,852(65.81 \%)$ \\
Schizophrenia diagnosis, $n$ (\%) & $73,653(30.48 \%)$ & $1139(4.30 \%)$ & $33,994(20.18 \%)$ \\
Bipolar disorder diagnosis, $n$ (\%) & $4007(1.66 \%)$ & $3482(13.14 \%)$ & $51,993(30.87 \%)$ \\
Major depressive disorder diagnosis, $n(\%)$ & $70,077(29.00 \%)$ & $15,535(58.62 \%)$ & $78,249(46.45 \%)$ \\
Diabetes diagnosis, $n$ (\%) & $151,333(62.63 \%)$ & $6200(23.39 \%)$ & $25,512(15.15 \%)$ \\
Hypertension diagnosis, $n$ (\%) & $21,365(8.84 \%)$ & $14,783(55.78 \%)$ & $45,069(26.76 \%)$ \\
Number of Charlson comorbidities, mean & $0.30(0.68)$ & $1.43(1.51)$ & $0.60(1.00)$ \\
$\quad(S D)$ & $45,197(18.70 \%)$ & $978(3.69 \%)$ & $24,808(14.73 \%)$ \\
Drug abuse, $n$ (\%) & $24,525(10.15 \%)$ & $794(3.00 \%)$ & $14,279(8.48 \%)$ \\
Alcoholism, $n(\%)$ &
\end{tabular}

$S D$ standard deviation 


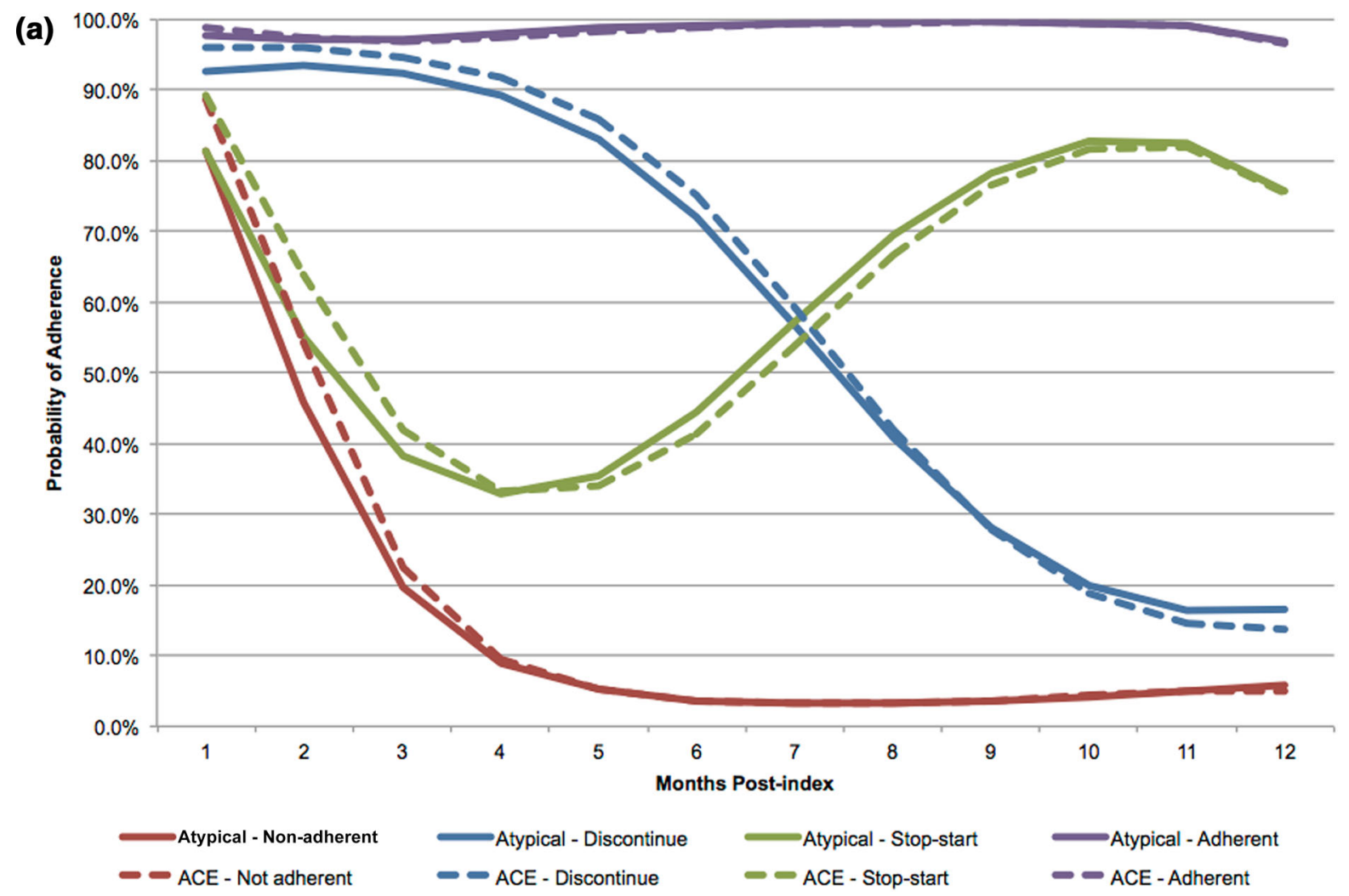

(b) $100.0 \%$
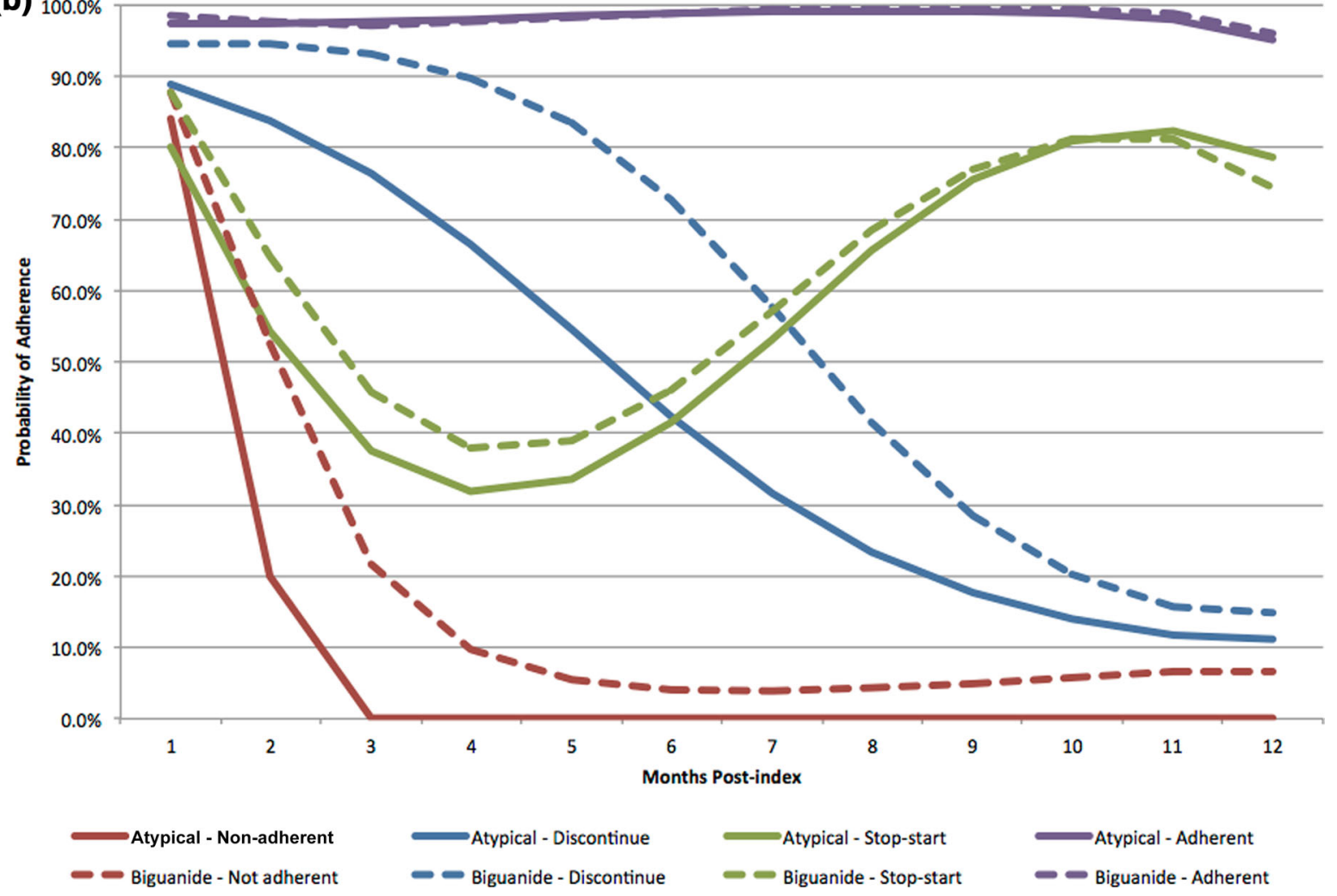


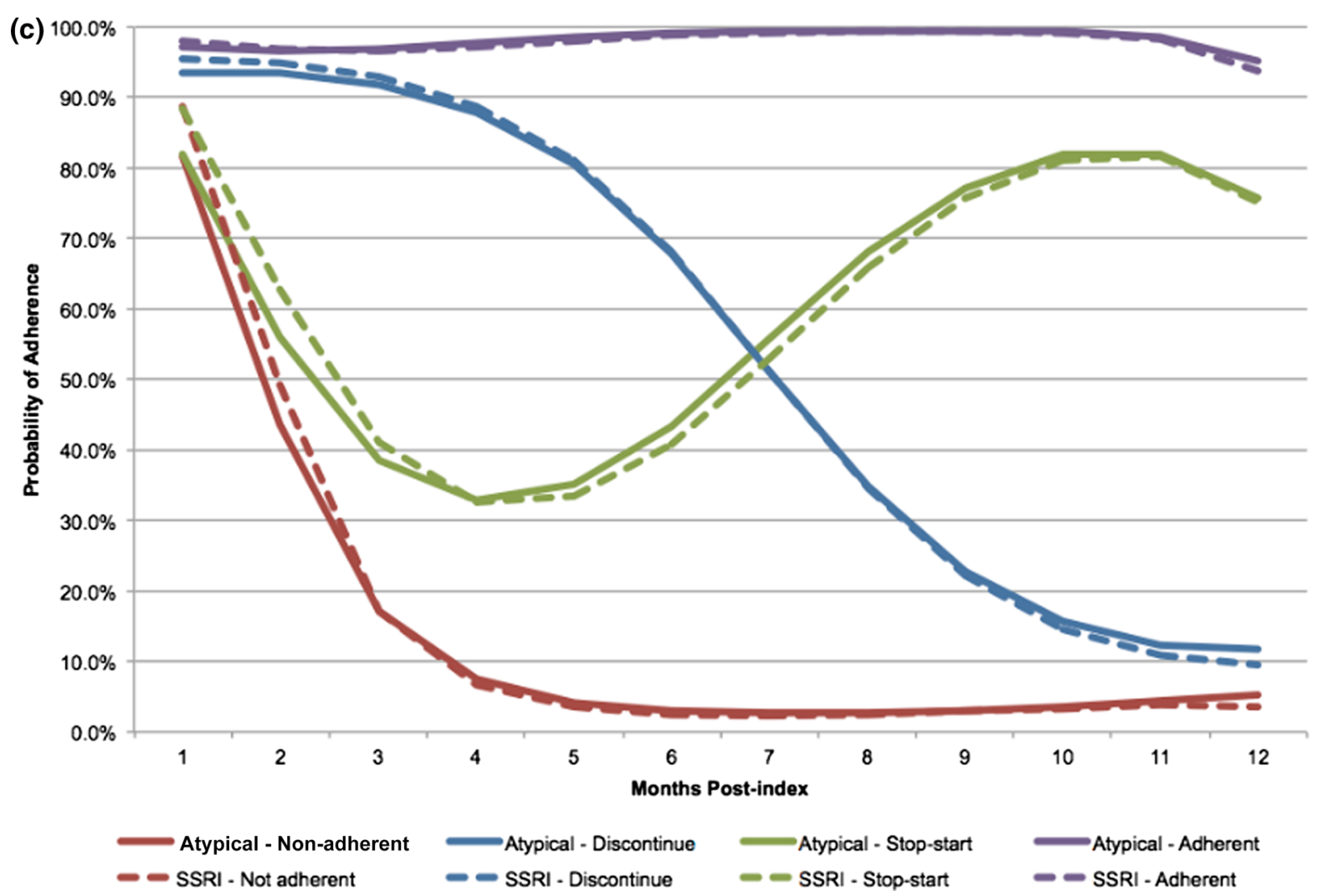

Fig. 1 continued

broke down as $29.2 \%$ non-adherent, $16.2 \%$ gradual discontinuers, $11.6 \%$ stop-start, and $43.0 \%$ adherent. The SSRI adherence distributions were $29.9 \%, 17.3 \%, 10.9 \%$, and $41.9 \%$ in the non-adherent, gradual discontinuation, stop-start, and adherent trajectory groups, respectively. Among those patients with an SMI and type 2 diabetes taking an atypical antipsychotic and a biguanide, the proportions of patients distributed across the adherence groups by medication were the most different. Antipsychotic adherence was $12.7 \%$ non-adherent, $20.5 \%$ gradual discontinuation, $11.3 \%$ stop-start, and 55.5\% adherent. In contrast, biguanide adherence was $23.3 \%$ non-adherent, $14.4 \%$ gradual discontinuation, $13.7 \%$ stopstart, and $48.5 \%$ adherent. Note that the antipsychotic adherence trajectories were different for each medication pair cohort as each cohort was comprised of different patients.

The share of patients who displayed the same adherence patterns for their atypical antipsychotic and other medicines was $44.5 \%$ for ACE inhibitors, $44.5 \%$ for biguanides, and $49.8 \%$ for SSRIs (Table 3 ). These figures imply that atypical antipsychotic adherence patterns provided a $19.5 \%, 19.5 \%$, and $24.6 \%$ increase (all $p<0.001$ ) in predictive ability, respectively, relative to random chance $(25 \%)$.

Further, the adherent and non-adherent trajectory patterns contributed the greatest predictive value compared to the stop-start or the gradual discontinuation groups. Patients adherent to their atypical antipsychotic were often adherent to their ACE inhibitor (59\%), biguanide (60\%), and SSRI (61\%). Among those who rapidly became non-adherent to their atypical antipsychotic, $40 \%$ of those on an ACE inhibitor, $37 \%$ of those taking biguanides, and $51 \%$ of those prescribed an SSRI were also rapidly non-adherent to their atypical antipsychotic. Patients that fell into gradual discontinuation for both medications were $21 \%$ for ACE inhibitors, $17 \%$ for biguanides, and 33\% 
Table 3 Predictive accuracy of atypical antipsychotic adherence patterns

\begin{tabular}{|c|c|c|c|c|}
\hline $\begin{array}{l}\text { Atypical } \\
\text { adherence group }\end{array}$ & $\begin{array}{l}\text { Other drug } \\
\text { adherence group }\end{array}$ & $\begin{array}{l}\text { ACE inhibitor } \\
\text { share within } \\
\text { atypical group (\%) }\end{array}$ & $\begin{array}{l}\text { Biguanides } \\
\text { share within } \\
\text { atypical group (\%) }\end{array}$ & $\begin{array}{l}\text { SSRI Share within } \\
\text { atypical group (\%) }\end{array}$ \\
\hline \multirow[t]{4}{*}{ Non-adherent } & Non-adherent & 40 & 37 & 51 \\
\hline & Gradual discontinuation & 14 & 13 & 15 \\
\hline & Stop-start & 12 & 16 & 9 \\
\hline & Adherent & 34 & 34 & 25 \\
\hline \multirow[t]{4}{*}{ Gradual discontinuation } & Non-adherent & 32 & 35 & 28 \\
\hline & Gradual discontinuation & 21 & 17 & 33 \\
\hline & Stop-start & 12 & 15 & 10 \\
\hline & Adherent & 35 & 32 & 30 \\
\hline \multirow[t]{4}{*}{ Stop-start } & Non-adherent & 28 & 28 & 27 \\
\hline & Gradual discontinuation & 14 & 15 & 16 \\
\hline & Stop-start & 21 & 24 & 27 \\
\hline & Adherent & 36 & 33 & 30 \\
\hline \multirow[t]{4}{*}{ Adherent } & Non-adherent & 19 & 16 & 17 \\
\hline & Gradual discontinuation & 14 & 14 & 14 \\
\hline & Stop-start & 9 & 11 & 7 \\
\hline & Adherent & 59 & 60 & 61 \\
\hline \multicolumn{2}{|l|}{ Accuracy } & 44.5 & 44.5 & 49.6 \\
\hline \multicolumn{2}{|c|}{ Difference from random accuracy (25\%) } & 19.5 & 19.5 & 24.6 \\
\hline \multicolumn{2}{|c|}{$p$ value } & $<0.001$ & $<0.001$ & $<0.001$ \\
\hline
\end{tabular}

Non-adherent PDC $<80 \%$; Adherent PDC $\geq 80 \%$

for SSRIs. The stop-start group for both medications was $21 \%$ of ACE inhibitors, $24 \%$ of biguanides, and $27 \%$ of SSRIs. The trajectory pattern predictive accuracies for atypical antipsychotics and SSRIs were consistently greater than the anti-diabetes and anti-hypertension medications.

\section{Predictive Ability of Antipsychotic Adherence versus Patient Characteristics}

Medication adherence predictions were more accurate when they were based on atypical antipsychotic patterns rather than patient characteristics alone. $R$-squared values were highest for adherence patterns and patient characteristics together, followed by adherence patterns alone (Fig. 2). This finding was confirmed across all medications and adherence groups except the biguanides discontinue and stop-start groups, where the $R$-squared values were approximately equal (discontinue: atypical trajectory $R$-squared and atypical trajectory plus characteristics $R$-squared $=0.002$; stop-start: atypical trajectory $R$-squared and atypical trajectory plus characteristics $R$-squared $=0.017$ ). 

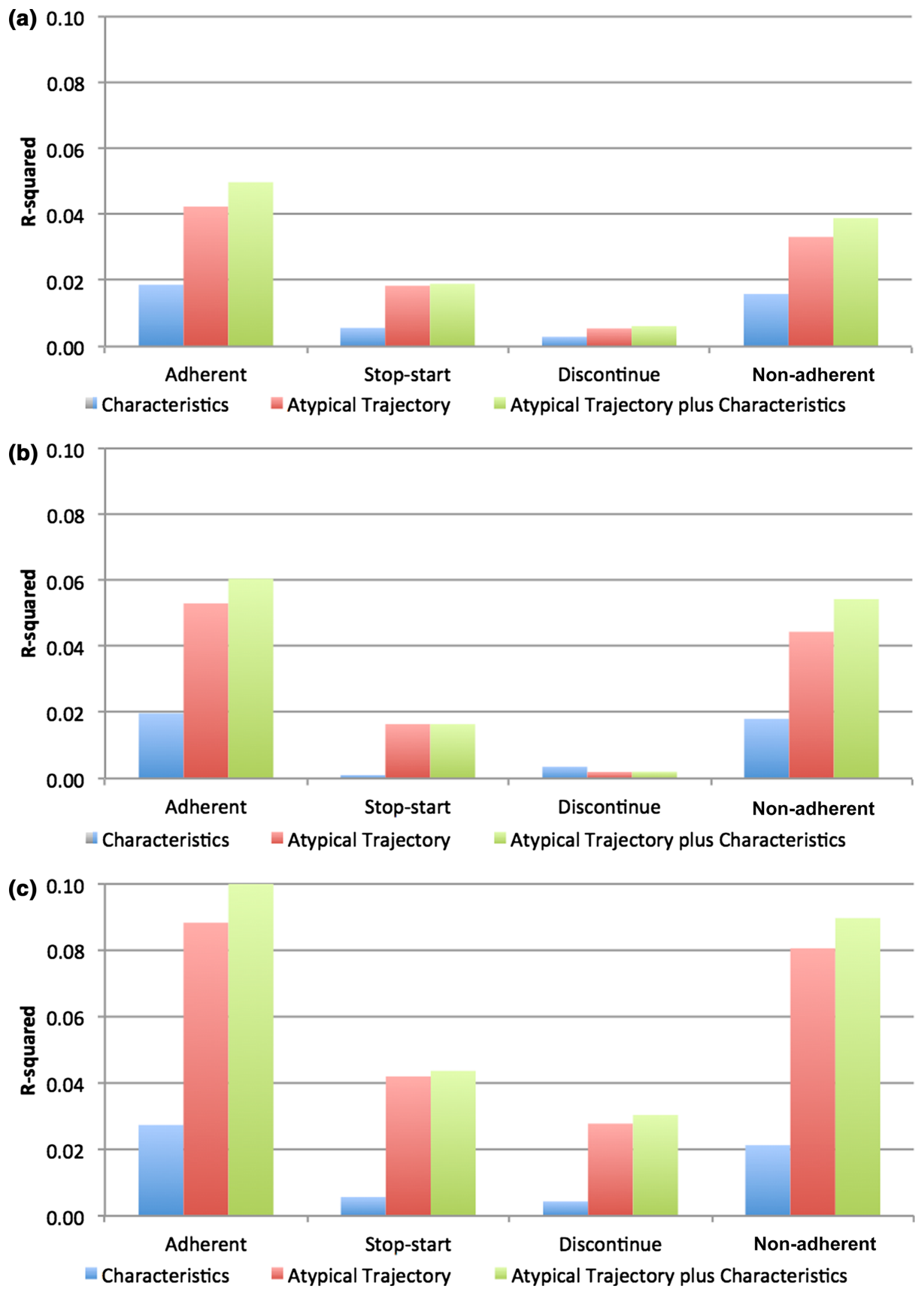
4Fig. 2 Ability of adherence trajectory to atypical antipsychotics vs. patient characteristics in predicting adherence trajectories to (a) ACE inhibitors, (b) biguanides, and (c) SSRIs

\section{Sensitivity Analysis}

We conducted a sensitivity analysis with three adherence groups. The patterns observed represented cases where (1) the patient rapidly became non-adherent, (2) the patient gradually discontinued therapy, and (3) the patient remained adherent throughout the 12-month period. Similar results to the primary analysis appeared in the sensitivity analysis. Details of these results can be found in the Online Resources (Figure A1, Table A2, Figure and A2).

\section{DISCUSSION}

This study found that adherence patterns to atypical antipsychotics could improve the ability to predict adherence patterns to ACE inhibitors, biguanides, and SSRIs among patients with serious mental and physical illnesses. The group trajectory model identified four trajectory groups: non-adherent, patients who exhibited discontinuation quickly after treatment; gradual discontinuation, those who discontinued more gradually over the 12-month study period; stop-start, patients who stopped treatment after a few months and later restarted; and adherent, a group that was largely adherent during the study period. Patients who were adherent to their atypical antipsychotic or non-adherent to their antipsychotic were more likely to be adherent or non-adherent, respectively, to their other medication, compared to those who discontinued or stopped and started again. Compared to random accuracy, patterns of atypical adherence are better predictors of adherence to ACE inhibitors and biguanides by $19.5 \%$ each and to SSRIs by $24.6 \%$ (all $p<0.001$ ). Similar patterns of adherence have been previously identified in patients prescribed an oral atypical antipsychotic and diagnosed with schizophrenia [26]. These trajectory groups highlight the inadequacies of metrics that calculate adherence as an average over a fixed time period (e.g., PDC). The nuances of the discontinuation and the stop-start adherence groups are not captured in a PDC metric or pill count over the course of multiple months.

Additionally, information on patient adherence patterns provides greater predictive ability than patient characteristics alone. Adherence patterns and patient characteristics combined had the highest $R$-squared values across all medications and trajectory groups, except in the discontinuing and stop-start biguanides users, where the $R$-squared of atypical antipsychotic adherence alone and with patient characteristics were equal. $R$-squared values ranged from 0.001 for characteristics alone among those prescribed biguanides in the stop-start category to 0.090 for both adherence to atypical antipsychotics and characteristics among those adherent to their SSRIs. Opolka et al. reported a similar range of the predictive ability of patient ethnicity and medications in predicting adherence to antipsychotic medications [48]. Their adjusted $R$-squared value for a model without either of these variables was 0.059 , increasing to 0.066 with ethnicity only, 0.091 with medication only, and 0.096 with both ethnicity and medication variables. Similar to the findings in our study, these results suggest that information about adherence to antipsychotic medications is important for understanding and predicting a patient's adherence to other medications. The combined predictive power of a patient's adherence to their atypical and the patient's characteristics may have clinical implications to help providers anticipate their patients' behaviors and create appropriate and individualized treatment plans.

New technologies like digital medicine offer the ability to track true adherence to oral medications and create more refined trajectories $[49,50]$. In the short run, however, these technologies may be available only for a limited set of medications, and their utility for patients with multiple chronic conditions may be unclear. This study shows that information on a patient's atypical antipsychotic adherence trajectory can provide useful predictive value for a patient's adherence patterns to their other 
medication. A model of adherence utilizing information about a patients' adherence pattern to an atypical antipsychotic provides more informative predictions than one relying solely on patient characteristics available in claims data. Thus, clinicians, case managers, payers, and policymakers may wish to use information on patients' prior adherence patterns to target adherence improvement programs to those patients most likely to be non-adherent based on their previous medication adherence.

Our study had several limitations. First, there may be more than four trajectory groups in practice. Sample size limitations, however, restricted our ability to observe additional patterns. For example, if we identified five adherence groups, we would have 25 predictive groups for each medication pair of interest and these additional comparisons limit the power of each predictive group. Second, our $R$-squared values were relatively low. Nevertheless, these $R$-squared values were in line with previous literature predicting adherence based on ethnicity and other characteristics that may impact adherence [48]. Third, the number of variables used in the model was not accounted for when measuring prediction accuracy. The atypical adherence model had fewer independent variables than the one for patient characteristics alone. Thus, the discrepancy between predictive power using a metric such as the Akaike information criterion would have been even larger than what was reported based on the $R$-squared metric [51]. Fourth, claims data do not accurately measure adherence because they capture medication purchase, but not ingestion, and do not contain information on reasons for discontinuation, (e.g., adverse events, lack of efficacy). This limitation may be mitigated in the future as technological devices to track true adherence in real time are developed. Fifth, adherence in patients newly initiating medications may not mirrored adherence among established patients. A similar study will be required in a population of patients who are not new to oral medications to treat these conditions. Sixth, patients who did not fill their initial atypical antipsychotic prescription were omitted from our patient sample. Therefore, the adherence patterns of the least adherent patients are not captured in our analysis. Further, there may be other factors not accounted for in this study that may also explain adherence patterns, such as a patient's pill burden beyond SMI, anti-diabetes, and anti-hypertension medications. Finally, the results of this study are particular to the medications included in the analysis and may not be generalizable to other medications, populations, or disease states.

\section{CONCLUSIONS}

Among patients with multiple chronic mental and physical illnesses, patterns of atypical antipsychotic adherence were useful predictors of adherence patterns to a patient's adherence to ACE inhibitors, biguanides, and SSRIs. Information on patient adherence patterns to atypical antipsychotics was a better predictor of patient adherence to these three medications than would be the case using patient demographic and clinical characteristics alone.

\section{ACKNOWLEDGEMENTS}

Funding. Funding for this study, journal processing charges, and the Open Access fee were provided by Otsuka Pharmaceutical Development \& Commercialization, Inc.

Authorship. All named authors meet the International Committee of Medical Journal Editors (ICMJE) criteria for authorship for this article, take responsibility for the integrity of the work as a whole, and have given their approval for this version to be published. Joanna P. MacEwan and Jason Shafrin had full access to all of the data in this study and take complete responsibility for the integrity of the data and accuracy of the data analysis.

Disclosures. Joanna P. MacEwan is an employee of Precision Health Economics, a healthcare consulting firm that received funding for this study from Otsuka Pharmaceutical Development \& Commercialization, Inc. Alison 
R. Silverstein is an employee of Precision Health Economics. Jason Shafrin is an employee of Precision Health Economics. Darius N. Lakdawalla is a consulting Scientific Advisor to Precision Health Economics and an investor in its parent company, Precision Medicine Group. Ainslie Hatch is an employee of Otsuka America Pharmaceutical, Inc. Felicia M. Forma is an employee of Otsuka Pharmaceutical Development \& Commercialization, Inc.

Compliance with Ethics Guidelines. This article does not contain any studies with human participants or animals performed by any of the authors.

Data Availability. The datasets generated during and/or analyzed during the current study are not publicly available because they are derived from proprietary Truven MarketScan ${ }^{\circledR}$ databases.

Open Access. This article is distributed under the terms of the Creative Commons Attribution-NonCommercial 4.0 International License (http://creativecommons.org/licenses/ by-nc/4.0/), which permits any noncommercial use, distribution, and reproduction in any medium, provided you give appropriate credit to the original author(s) and the source, provide a link to the Creative Commons license, and indicate if changes were made.

\section{REFERENCES}

1. National Institute of Mental Health. Serious Mental Illness (SMI) Among US Adults. Accessed 10, Jul 2017]; Available from: https://www.nimh.nih.gov/ health/statistics/prevalence/serious-mental-illnesssmi-among-us-adults.shtml.

2. Carney CP, Jones LE. Medical comorbidity in women and men with bipolar disorders: a population-based controlled study. Psychosomatic Med. 2006;68(5):684-91.

3. Dixon L, et al. The association of medical comorbidity in schizophrenia with poor physical and mental health. J Nervous Mental Dis. 1999;187(8):496-502.
4. Henderson DC. Schizophrenia and comorbid metabolic disorders. J Clin Psychiatry. 2005;66(Suppl 6):11-20.

5. Kilbourne AM, et al. Cardiovascular disease and metabolic risk factors in male patients with schizophrenia, schizoaffective disorder, and bipolar disorder. Psychosomatics. 2007;48(5):412-7.

6. Kilbourne AM, et al. Burden of general medical conditions among individuals with bipolar disorder. Bipolar Disord. 2004;6(5):368-73.

7. Hert M, et al. Physical illness in patients with severe mental disorders. I. Prevalence, impact of medications and disparities in health care. World Psychiatry. 2011;10(1):52-77.

8. Sachs GS, et al. Rationale, design, and methods of the systematic treatment enhancement program for bipolar disorder (STEP-BD). Biol Psychiatry. 2003;53(11):1028-42.

9. Sendt K-V, Tracy DK, Bhattacharyya S. A systematic review of factors influencing adherence to antipsychotic medication in schizophrenia-spectrum disorders. Psychiatry Res. 2015;225(1):14-30.

10. Dolder CR, Lacro JP, Jeste DV. Adherence to antipsychotic and nonpsychiatric medications in middle-aged and older patients with psychotic disorders. Psychosomatic Med. 2003;65(1):156-62.

11. Velligan DI, et al. Adherence problems in patients with serious and persistent mental illness. J Clin Psychiatry. 2009;70(suppl 4):1-48.

12. Ayuso-Gutiérrez JL, del Río JM. Vega, Factors influencing relapse in the long-term course of schizophrenia. Schizophrenia Res. 1997;28(2):199-206.

13. Hirsch S, et al. A one year prospective study of the effect of life events and medication in the aetiology of schizophrenic relapse. $\mathrm{Br} \mathrm{J}$ Psychiatry. 1996;168(1):49-56.

14. Gilmer TP, et al. Adherence to treatment with antipsychotic medication and health care costs among Medicaid beneficiaries with schizophrenia. Am J Psychiatry. 2004;161(4):692-9.

15. Offord S, et al. Impact of oral antipsychotic medication adherence on healthcare resource utilization among schizophrenia patients with Medicare coverage. Community Mental Health J. 2013;49(6):625-9.

16. Valenstein $\mathrm{M}$, et al. Pharmacy data identify poorly adherent patients with schizophrenia at increased risk for admission. Med Care. 2002;40(8):630-9. 
17. Weiden PJ, et al. Partial compliance and risk of rehospitalization among California Medicaid patients with schizophrenia. Psychiatric Serv. 2004;55(8):886-91.

18. Currie CJ, et al. The impact of treatment noncompliance on mortality in people with type 2 diabetes. Diabetes Care. 2012;35(6):1279-84.

19. Fenn HH, et al. Medical comorbidity and healthrelated quality of life in bipolar disorder across the adult age span. J Affective Disord. 2005;86(1):47-60.

20. Insel, T.R., Assessing the economic costs of serious mental illness. Am J Psychiatry, 2008;165(6):663665.

21. Osterberg L, Blaschke T. Adherence to medication. N Engl J Med. 2005;353(5):487-97.

22. Lam WY, Fresco P. Medication adherence measures: an overview. Biomed Res Int. 2015;2015:217047.

23. Hess LM, et al. Measurement of adherence in pharmacy administrative databases: a proposal for standard definitions and preferred measures. Ann Pharmacother. 2006;40(7-8):1280-8.

24. Leslie SR, et al. Calculating medication compliance, adherence and persistence in administrative pharmacy claims databases. Pharmaceutical Program. 2008;1(1):13-9.

25. National Committee for Quality Assurance. Adherence to antipsychotic medication for people with schizophrenia. 2015 [cited 2017 September 6]; Available from: http://www.ncqa.org/report-cards/ health-plans/state-of-health-care-quality/2015-tabl eof-contents/antipsychotic-medications.

26. MacEwan JP, et al. Patterns of adherence to oral atypical antipsychotics among patients diagnosed with schizophrenia. J Managed Care Specialty Pharm. 2016;22(11):1349-61.

27. Bangsberg DR. Monitoring adherence to HIV antiretroviral therapy in routine clinical practice: the past, the present, and the future. AIDS Behav. 2006;10(3):249.

28. Garber MC, et al. The concordance of self-report with other measures of medication adherence: a summary of the literature. Med Care. 2004;42(7):649-52.

29. Stephenson J, et al. Adherence to oral second-generation antipsychotic medications in patients with schizophrenia and bipolar disorder: physicians' perceptions of adherence vs. pharmacy claims. Int J Clin Pract. 2012;66(6):565-73.
30. Karve S, et al. Prospective validation of eight different adherence measures for use with administrative claims data among patients with schizophrenia. Val Health. 2009;12(6):989-95.

31. Carney CP, Jones L, Woolson RF. Medical comorbidity in women and men with schizophrenia: a population-based controlled study. J Gen Internal Med. 2006;21(11):1133-7.

32. Kamat SA, et al. Prevalence and humanistic impact of potential misdiagnosis of bipolar disorder among patients with major depressive disorder in a commercially insured population. J Managed Care Pharm: JMCP. 2008;14(7):631-42.

33. Quan $\mathrm{H}$ et al. Coding algorithms for defining comorbidities in ICD-9-CM and ICD-10 administrative data. Med Care, 2005: p 1130-1139.

34. Lee TA, et al. Osteoarthritis: a comorbid marker for longer life? Ann Epidemiol. 2007;17(5):380-4.

35. Novartis Pharmaceuticals Corporation. Highlights of Prescrining Information: CLOZARIL ${ }^{\circledR}$ (clozapine) tablets. 2014 [cited 2015 Aug 17].

36. Patel NC et al. Drug adherence: effects of decreased visit frequency on adherence to clozapine therapy. Pharmacotherapy: The Journal of Human Pharmacology and Drug Therapy, 2005;25(9):1242-1247.

37. Practice Guideline for the Treatment of Patients with Bipolar Disorder. 2002, American Psychiatric Association.

38. Practice Guideline for the Treatment of Patients with Schizophrenia. 2004, American Psychiatric Association.

39. Practice Guideline for the Treatment of Patients with Major Depressive Disorder. 2010, American Psychiatric Association.

40. Mental Health Medications. 2016 January 2016 2/11/2016]; Available from: http://www.nimh.nih. gov/health/topics/mental-health-medications/ind ex.shtml\#part_149866.

41. Agency for Healthcare Research and Quality, Treatment for Bipolar Disorder, in Evidence-based Practice Center Systematic Review Protocol, Effective Health Care Program. June 2014.

42. Prevention or Delay of Type. 2 Diabetes. Diabetes Care. 2016;39(Suppl 1):S36-8.

43. Bennett WL et al., Oral diabetes medications for adults with type 2 diabetes: an update, in Comparative Effectiveness Review. 2011, Agency for Healthcare Research and Quality: Rockville, MD. 
44. James PA, et al. 2014 evidence-based guideline for the management of high blood pressure in adults: report from the panel members appointed to the eighth joint national committee (jnc 8). JAMA. 2014;311(5):507-20.

45. Weber MA, et al. Clinical practice guidelines for the management of hypertension in the community. J Clin Hypertension. 2014;16(1):14-26.

46. National Quality Measures, C., Adherence to antipsychotic medications for individuals with schizophrenia: percentage of members 19 to 64 years of age during the measurement year with schizophrenia who were dispensed and remained on an antipsychotic medication for at least 80 percent of their treatment period. 2015.

47. Nau DP. Proportion of days covered (PDC) as a preferred method of measuring medication adherence. Springfield: Pharmacy Quality Alliance; 2012.
48. Opolka JL, et al. Role of ethnicity in predicting antipsychotic medication adherence. Ann Pharmacother. 2003;37(5):625-30.

49. Profit D, et al. Developing a digital medicine system in psychiatry: ingestion detection rate and latency period. J Clin Psychiatry. 2016;77(9):1095-100.

50. Rohatagi S, et al. Optimization of a digital medicine system in psychiatry. J Clin Psychiatry. 2016;77(9):1101-7.

51. Bozdogan H. Model selection and Akaike's information criterion (AIC): the general theory and its analytical extensions. Psychometrika. 1987;52(3):345-70. 